Les réacteurs de fusion

imposeront aux matériaux

situés face au plasma de forts flux de neutrons rapides,

ainsi que des flux thermiques et des températures très élevés. Seuls des matériaux de structure cristalline adéquate, dont

la composition chimique et la microstructure auront été conçues à dessein, pourront résister à ces conditions de service.

Dans la perspective de la construction d'un réacteur de démonstration vers le milieu de ce siècle, on présente ici quelques points cruciaux de l'effort de recherche et de développement de nouveaux matériaux capables de résister aux conditions imposées.

Puis, on souligne la nécessité d'un programme ambitieux de validation et qualification de ces matériaux.

\title{
Réacteurs de fusion : le défi majeur des matériaux
}

Jean-Louis Boutard (jean-louis.boutard@cea.fr)

Cabinet du Haut-Commissaire à l'énergie atomique, CEA/Saclay, 91191 Gif-sur-Yvette Cedex

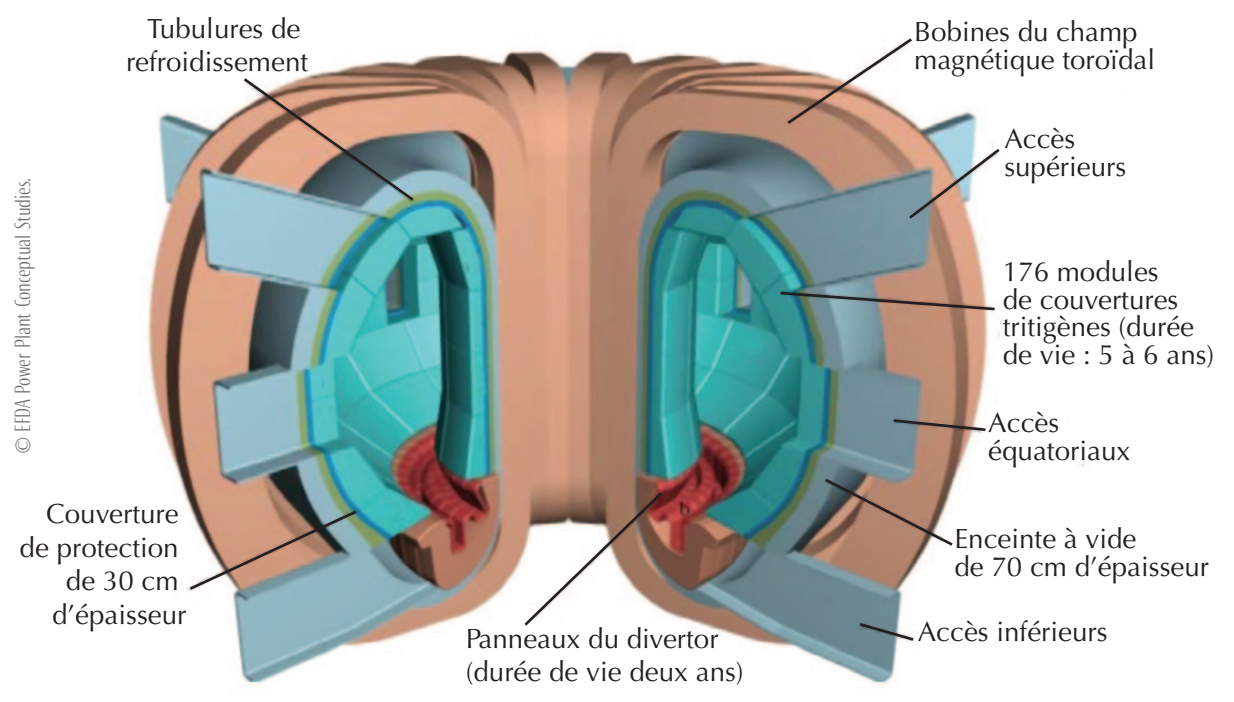

1. Schéma d'un réacteur tokamak étudié dans le cadre des Power Plant Conceptual Studies de l'EFDA [3]. Les puissances électrique et de fusion sont respectivement de 1,45 et 3,41 GW. Le grand rayon et la hauteur de l'enceinte à vide sont de l'ordre de $10 \mathrm{~m}$. La proposition de segmentation des couvertures tritigènes donne une idée de l'agencement interne. Les durées de vie sont indicatives. Concernant le système magnétique, seules figurent les bobines du champ toroïdal (6 teslas). La première paroi, qui protège les couvertures face au plasma, n’a pas été dessinée.

Dans les réacteurs de fusion, l'énergie est fournie par la réaction nucléaire entre le deutérium $\left({ }^{2} \mathrm{H} \equiv \mathrm{D}\right)$ et le tritium $\left({ }^{3} \mathrm{H} \equiv \mathrm{T}\right)$, deux isotopes de l'hydrogène [1] : ${ }^{2} \mathrm{H}+{ }^{3} \mathrm{H} \rightarrow{ }^{4} \mathrm{He}(3,56 \mathrm{MeV})+$

${ }^{1} \mathrm{n}(14,03 \mathrm{MeV})$ (1).

Les neutrons de $14 \mathrm{MeV}$ issus de la réaction de fusion D-T (1) sont ralentis par les matériaux des couvertures tritigènes ; leur énergie cinétique est ainsi transformée en énergie thermique, qui est évacuée par le fluide de refroidissement. Dans les futurs réacteurs de fusion, cette énergie sera utilisée pour produire de l'électricité.

Le projet ITER permettra de répondre à de nombreuses questions scientifiques et technologiques relatives à la filière tokamak, mais la fluence neutronique y sera trop faible pour poser des problèmes d'effets d'irradiation dans les matériaux. En revanche, pour les futurs réacteurs de fusion, la résistance à de fortes températures de service, à des flux thermiques très élevés et aux dégâts d'irradiation, constituera un défi majeur.

Les différents partenaires d'ITER, dont l'Europe, anticipent la construction, d'ici le milieu de ce siècle, d'un réacteur, communément appelé DEMO, démontrant la faisabilité de la production d'électricité à partir de la réaction de fusion DT (1) (voir encadré). Disposer de matériaux capables de supporter les conditions de service d'un tel réacteur constitue actuellement un verrou, qui ne pourra être levé que par un programme ambitieux de recherche et développement de nouveaux matériaux, dont nous allons esquisser, ci-dessous, quelques points importants.

Dans l'hypothèse d'un confinement magnétique du plasma de type tokamak, le plus étudié à ce jour, les composants des futurs réacteurs qui seront soumis aux 
Image virtuelle de l'intérieur du tokamak Tore Supra au CEA/Cadarache, tel qu'il sera dans sa nouvelle configuration WEST, avec le divertor constitué d’éléments similaires à ceux qui seront installés sur ITER.

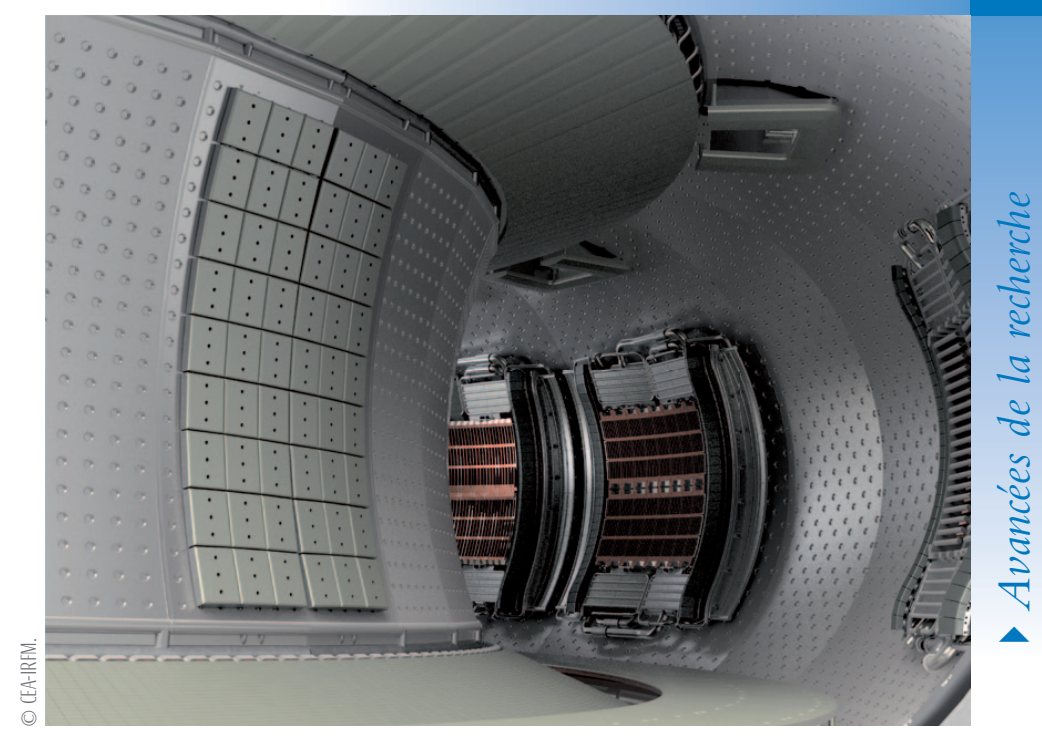

\section{Les composants internes d'un réacteur tokamak (voir figure 1)}

La chambre à vide d'un réacteur tokamak (fig. 1) est une enceinte toroïdale en acier inoxydable, complétée par un « divertor ». Les éléments de couvertures tritigènes sont accrochés sur la face interne de l'enceinte. Ils présentent, face au plasma, une première paroi de protection. Les flux thermiques que reçoivent ces structures imposeront qu'elles soient activement refroidies pour maitriser leur température. Les réactions de fusion (1) ont lieu dans le plasma confiné par la configuration magnétique tokamak dans l'espace torique limité par la première paroi et le divertor.

Dans un réacteur, la première paroi sera constituée d'une structure activement refroidie, d'épaisseur centimétrique, en acier martensitique protégé par du tungstène [4].

Le divertor est un dispositif raccordé à la partie inférieure de la chambre à vide. Il assure (i) l'extraction de la puissance emportée par les particules échappant au confinement magnétique, et (ii) le pompage de ces particules, notamment l'hélium qui constitue la " cendre » de la réaction de fusion et qui étoufferait la production d'énergie de fusion s'il s'accumulait dans le plasma. Le divertor d'ITER (fig. E1) sera constitué d'une structure en acier inoxydable maintenant des tubes en alliage de cuivre (Cu-Cr-Zr) refroidis à l'eau et protégés par des tuiles de tungstène. Les maquettes réalisées et testées montrent que ce dispositif devrait pouvoir extraire un flux thermique maximal de $\sim 10$ à $20 \mathrm{MW} / \mathrm{m}^{2}$. Dans le cadre du projet WEST, ce divertor sera fabriqué en condition industrielle représentative d'ITER, puis installé et testé à Cadarache dans Tore Supra (CEA/DSM/IRFM) (voir figure ci-dessus) [5] .

Le passage à un divertor de réacteur sera une tâche difficile. En effet, la puissance reçue par ce dispositif est, à fraction de puissance rayonnée par le plasma donnée, proportionnelle à la puissance de fusion. Toutes choses égales par ailleurs, passer de la puissance de fusion d'ITER $(0,5 \mathrm{GW})$ à une valeur typique de $3 \mathrm{GW}$ pour un réacteur, conduit à multiplier par quatre la puissance devant être extraite par le divertor, dans l'hypothèse où la surface active du divertor d'un réacteur de fusion est 1,5 fois plus grande que celle $d^{\prime}$ ITER. La tenue à des flux de 40 à $80 \mathrm{MW} / \mathrm{m}^{2}$ ne pourra pas reposer que sur des progrès technologiques, des matériaux ou des concepts plus avancés. L'atténuation des flux thermiques eux-mêmes, en étalant la surface d'impact des particules ou en augmentant la puissance rayonnée à travers toute la surface extérieure du plasma, parait nécessaire pour retrouver des flux thermiques raisonnables sur le divertor d'un réacteur.

Les couvertures tritigènes sont des composants nouveaux dans le monde de la fusion. Elles ont une triple fonction liée au caractère nucléaire du réacteur : (i) récupérer l'énergie cinétique des neutrons de $14 \mathrm{MeV}$ produits par la réaction de fusion (1) et la transformer en chaleur, transférée vers l'extérieur par un fluide de refroidissement; (ii) produire du tritium par réaction nucléaire ; (iii) protéger l'enceinte à vide et les dispositifs extérieurs (notamment les bobines supraconductrices qui créent le champ magnétique) contre les neutrons rapides et les rayonnements issus du plasma. Le tritium est produit dans les couvertures grâce à un matériau tritigène contenant du lithium, sous forme de céramique (orthosilicate $\mathrm{Li}_{4} \mathrm{SiO}_{4}$, sélectionné pour sa bonne stabilité thermique et chimique) ou d'eutectique liquide $\mathrm{Li}_{17}-\mathrm{Pb}_{83}$, pour permettre, en utilisant le neutron de la fusion D-T (1), la réaction suivante avec l'isotope ${ }^{6} \mathrm{Li}(6,5 \%$ du lithium naturel) : ${ }^{6} \mathrm{Li}+{ }^{1} \mathrm{n} \rightarrow \mathrm{T}+{ }^{4} \mathrm{He}+4,78 \mathrm{MeV}$.

Cette fonction est essentielle pour assurer l'autosuffisance en tritium, en produisant un noyau de tritium chaque fois que l'on en détruira un par fusion. Différents concepts de couverture, dont les structures sont en acier martensitique à activation réduite [2], seront testés dans ITER. L'Europe testera pour sa part deux concepts de référence ; I'un, développé par le Karlsruhe Institüt für Technologie (KIT), utilise des céramiques à base de lithium, I'autre, développé par le CEA, I'eutectique Li-Pb. Dans les deux types de couverture envisagés, des noyaux multiplicateurs de neutrons permettront de compenser les pertes inévitables : le béryllium pour la couverture à base de céramique, le plomb de l'eutectique Li-Pb dans le second cas. Dans les deux concepts, le refroidissement est assuré par de l'hélium gaz sous 80 bars.

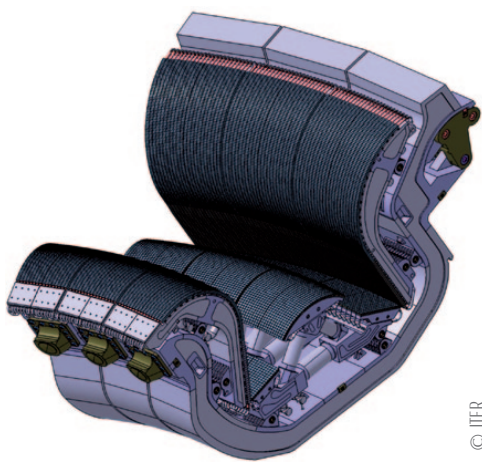

E1. Un élément du divertor d'ITER, composé d'une structure de soutien, essentiellement constituée d'acier inoxydable, et d'éléments en tungstène, face au plasma. 


\section{\〉}

conditions de service les plus sévères seront ceux situés face au plasma (voir encadré et fig. 1) : la première paroi et le divertor, puis les couvertures tritigènes. Pour situer les ordres de grandeur, le divertor sera soumis à des températures supérieures à $1000^{\circ} \mathrm{C}$, et à des flux thermiques de l'ordre de 10 à $20 \mathrm{MW} / \mathrm{m}^{2}$. La dose d'irradiation maximale visée pour la première paroi correspond à 50 à 80 dpa (déplacements par atome), ce qui est du même ordre de grandeur que pour les éléments combustibles d'un réacteur de fission à neutrons rapides, d'énergie $\sim 1 \mathrm{MeV}$. Mais, pour un matériau donné, les taux de transmutation induits par les neutrons de $14 \mathrm{MeV}$ de la fusion DT seront de plusieurs ordres de grandeur supérieurs, engendrant des concentrations élevées d'hydrogène et d'hélium pouvant fragiliser le matériau, et portant le risque de produire des isotopes radioactifs à vie longue [2].

Les matériaux de structure et de protection sélectionnés devront posséder une bonne fabricabilité, une bonne compatibilité avec les isotopes d'hydrogène et les fluides de refroidissement, une résistance mécanique significative à haute température, et pouvoir résister à des flux thermiques importants et aux dégâts d'irradiation des neutrons rapides de $14 \mathrm{MeV}$. Des matériaux à "basse activation » sont en cours de développement [2]. $\mathrm{Ne}$ produisant pas d'isotopes radioactifs à vie longue, ils devraient pouvoir être manipulés sans précaution complexe après quelques centaines d'années, avantage décisif par rapport au nucléaire de fission.

\section{Les axes de recherche actuels}

Dans l'article précédent, on a montré que seuls des matériaux de structure cristalline adéquate, ayant des compositions chimiques et microstructures conçues à dessein, pourront résister aux conditions de service des réacteurs de fusion. Un projet de recherche intégré au sein du programme EURATOM-fusion et coordonné par l'EFDA (European Fusion Design Agreement) est dédié au développement de ces matériaux [6].

Ci-après, quelques aspects importants de cet effort de recherche seront illustrés : la nanostructuration des matériaux, la modélisation physique associée à la simulation de l'irradiation neutronique par des particules chargées et à la caractérisation physicochimique à l'échelle appropriée, ainsi que la problématique de l'interaction entre le plasma et le tungstène dans le cas du divertor.

\section{Nanostructuration des matériaux}

Nanostructurer un matériau signifie introduire une forte densité $\left(\sim 10^{23}\right.$ à $\left.10^{24} \mathrm{~m}^{-3}\right)$ de précipités de taille nanométrique, dont les interfaces avec la matrice seront des pièges pour éliminer les défauts ponctuels, avec comme conséquences la diminution du durcissement et une meilleure résistance au gonflement.

Les aciers ferritiques ou martensitiques renforcés par dispersion de particules nanométriques d'oxydes d'yttrium et de titane (ODS : Oxide dispersion strengthened) appartiennent à cette catégorie. La figure $3 \mathrm{c}$ de l'article précédent [2] montre clairement l'effet bénéfique sur le gonflement. Ces aciers ont aussi un durcissement moindre (fig. $4 \mathrm{~b}$ de la référence [2]), confirmant que les nombreuses interfaces sont des puits efficaces pour éliminer les défauts ponctuels ; ils présentent, de plus, une résistance mécanique accrue à haute température.

En ce qui concerne l'hélium, on a affaire à un problème classique d'impureté fragilisante, s'il y a ségrégation aux joints de grains. Le cas historique bien connu est celui du soufre qui ségrège facilement, conduisant à une fragilisation intergranulaire inacceptable des aciers ferritiques. La pratique sidérurgique est de le piéger au sein des grains, sous forme de sulfure de manganèse. C'est la même idée qui est poursuivie pour l'hélium en multipliant les interfaces à l'intérieur des grains, où les atomes d'hélium pourront être piégés. Pour fixer les ordres de grandeur, si on fait l'hypothèse qu'un acier ODS contient $\sim 10^{23} \mathrm{~m}^{-3}$ précipités d'un rayon moyen de $2 \mathrm{~nm}$ et que sa taille de grain est $\sim 10^{-6} \mathrm{~m}$, la surface des interfaces particule/ matrice sera de $\sim 10^{6} \mathrm{~m}^{2}$ par grain, à comparer à la surface de joint d'un grain $\sim 10^{-12} \mathrm{~m}^{2}$. On s'attend donc à ce que la nanostructuration des aciers ODS soit particulièrement efficace pour piéger l'hélium à l'intérieur des grains. De fait, les premières irradiations en double faisceau (production de déplacements atomiques et implantation simultanée d'hélium) indiquent que les interfaces entre la matrice et les dispersoïdes d'un acier ODS piègent efficacement les atomes d'hélium [7].

Ces aciers ferritiques ou martensitiques ODS, élaborés par métallurgie des poudres et mécano-synthèse, avaient fait l'objet de développements importants, notamment par la société INCO, à la fin des années 1970. Le marché visé était les gaines de l'élément combustible des réacteurs à neutrons rapides. L'absence de marché a conduit INCO puis Plansee à l'arrêt de la production. Un effort est actuellement en cours en Europe, et particulièrement en France, pour se réapproprier les procédés d'élaboration et de mise en forme de ces matériaux [8] et résoudre les problèmes de métallurgie physique que posent leur mécanosynthèse et l'échelle nanométrique de leur microstructure [9].

\section{Modélisation des effets d'irradiation et validation expérimentale par particules chargées}

La modélisation des effets macroscopiques de l'irradiation (évolution des microstructures et des propriétés mécaniques), qui est de nature multiéchelle, est devenue prédictive dans des alliages modèles, grâce aux données fournies par le calcul ab initio.

L'article [10] donne un aperçu des progrès considérables faits, pendant les dix dernières années, dans le domaine des calculs de structure électronique basés sur la Théorie de la Fonctionnelle de la Densité (DFT en anglais) et du rôle primordial joué par ces calculs pour déterminer les configurations stables des défauts ponctuels et de leurs amas, leurs énergies de formation et de migration, ainsi que le paysage énergétique vu par une dislocation lors de son glissement.

Par exemple, les calculs ab initio permettent de décrire les mécanismes de migration des lacunes et des interstitiels, qui contrôlent quantitativement la diffusion atomique dans les alliages métalliques sous vieillissement thermique (lacunes) ou sous irradiation (interstitiels), et donc l'évolution lente de leur microstructure (voir [11] pour le cas du fer).

Différentes méthodes cinétiques permettent ensuite de simuler l'évolution des alliages lors d'un vieillissement thermique ou sous irradiation. La méthode de Monte Carlo cinétique à l'échelle atomique est la plus précise, car elle simule l'enchaînement des sauts successifs des défauts et permet d'explorer les premiers stades de la précipitation. Cependant, bien que l'on puisse simuler jusqu'à $10^{9}$ sauts, les temps explorés restent courts (fig. 2). Pour des évolutions cinétiques plus longues, on doit utiliser des méthodes simplifiées. Dans la méthode de cinétique chimique homogène, le système est simplement décrit par les concentrations d'amas de défauts ponctuels et de précipités. On écrit que l'évolution de la taille de ces derniers au cours du temps résulte du bilan des flux d'atomes, contrôlés par la diffusion, se dirigeant vers les précipités ou s'en 


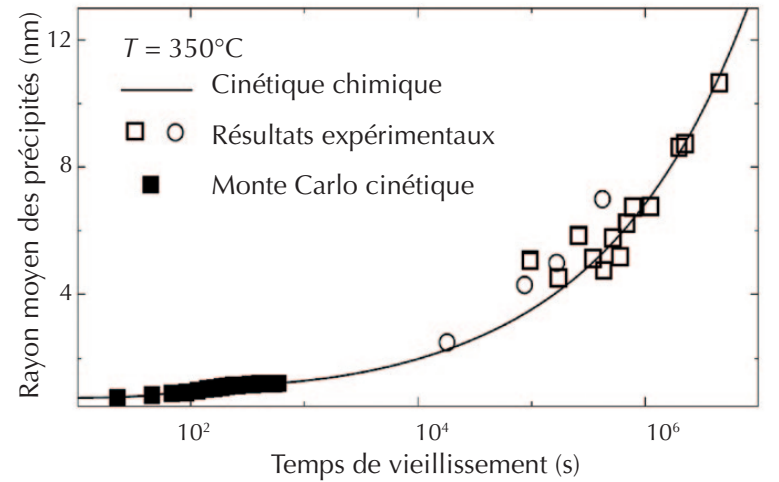

2. Cinétique d'évolution du rayon moyen des précipités de composition chimique $\mathrm{Al}_{3} \mathrm{Zr}$, lors d'un recuit à $350^{\circ} \mathrm{C}$ d'un alliage d'aluminium Al-Zr. La simulation Monte Carlo permet d'étudier les temps courts, jusque vers 1000 s, qui correspondent à la germination des précipités pour lesquels les points expérimentaux n'existent pas. (E. Clouet et al., Acta Mater., 53 (2005) 2313-2325.)
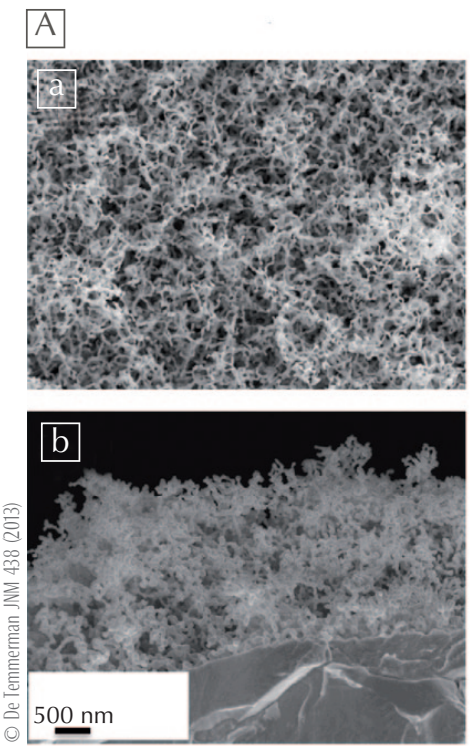

$\mathrm{B}$
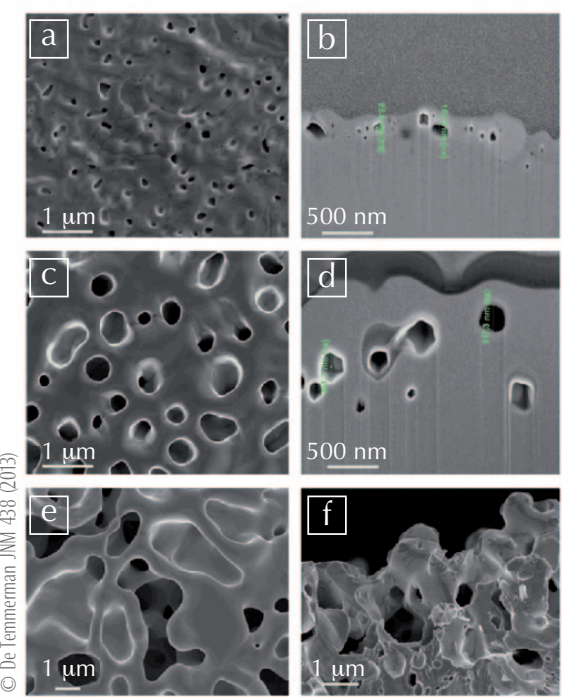

3. Simulation expérimentale de l'interaction plasma-tungstène sous des flux de chaleur supérieurs à $10 \mathrm{MW} / \mathrm{m}^{2}[15]$.

(A) Nanostructure obtenue sous irradiation de particules $\alpha$ de $50 \mathrm{eV}$ pendant $500 \mathrm{~s}$. La température de surface du tungstène était $\sim 1000^{\circ} \mathrm{C}$. (a) Vue de dessus ; (b) coupe verticale.

(B) Nanostructure obtenue avec des particules $\alpha$ de $40 \mathrm{eV}$ et une température de surface du tungstène de $\sim 2000^{\circ} \mathrm{C}$. Colonne de gauche : vue de dessus. Colonne de droite : vue en coupe. La taille des bulles croît avec le temps : $t=10 s(\mathrm{a})$ et (b) ; $t=30 \mathrm{~s}(\mathrm{c})$ et $(\mathrm{d}) ; \mathrm{t}=1000 \mathrm{~s}(\mathrm{e})$ et (f).

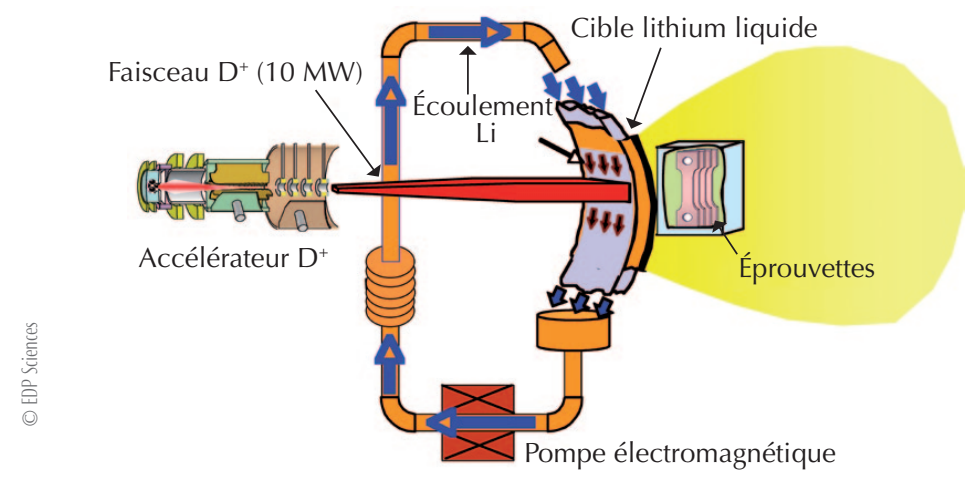

4. Schéma de principe de l'installation IFMIF. La longueur de l'ensemble dépassera $100 \mathrm{~m}$. (Source : J.L. Bobin, La fusion thermonucléaire contrôlée, EDP Sciences (2011)). évaporant. À titre d'exemple, la figure 2 présente le calcul de l'évolution de la taille des précipités dans un cas simple, tiré de l'aéronautique. Les simulations de cinétique chimique et Monte Carlo utilisent les mêmes paramètres calculés $a b$ initio et il n'y a aucun paramètre ajustable. L'accord de la cinétique chimique est excellent avec les prédictions Monte Carlo aux temps courts et avec les données expérimentales obtenues, pour des temps plus longs, par microscopie électronique à transmission et sonde atomique tomographique.

Les compositions chimiques et microstructures des alliages industriels sont beaucoup plus complexes que celles modélisées à ce jour. La prédiction des cinétiques de vieillissement sous irradiation nécessitera de bien discerner ce qu'il y a de déterminant dans cette complexité et d'introduire les mécanismes de diffusion par les interstitiels.

La Dynamique des dislocations discrètes (DDD) est un outil fiable pour prédire le comportement collectif d'un ensemble de dislocations en cours de déformation plastique et les limites d'élasticité qui en résultent. Elle consiste à écrire l'évolution de la population de dislocations qui interagissent entre elles par leur champ de contrainte à longue portée et sont soumises à la présence d'une contrainte mécanique extérieure appliquée [12]. L'interaction des dislocations avec les différents obstacles au glissement mis en place par l'irradiation est à l'origine du durcissement. Savoir décrire cette interaction est un chantier important qui débute et qui sera incontournable pour une modélisation prédictive du durcissement dans les décennies à venir.

Nous voudrions aussi souligner l'importance des implantations/irradiations par particules chargées, en étroite interaction avec l'effort de modélisation. L'implantation de particules $\alpha$ de quelques dizaines de $\mathrm{MeV}$ a permis la mise en évidence de l'effet fragilisant de l'hélium [2] et une première quantification de cet effet sur la contrainte de clivage et la ténacité [13]. Les irradiations par faisceaux d'ions de quelques $\mathrm{MeV}$, en configuration simple (dpa), double $(\mathrm{dpa}+\mathrm{He})$ ou triple faisceau $(\mathrm{dpa}+\mathrm{He}+\mathrm{H})$ [1], constituent des outils fiables flexibles et à retour d'expérience rapide pour simuler la production simultanée de déplacements atomiques et de produits de transmutation typiques de la fusion. Elles sont particulièrement bien adaptées aux études 


\section{$>>$}

paramétriques d'évolution microstructurale utilisant les moyens modernes de caractérisation physico-chimique jusqu'à l'échelle nanométrique, comme la microscopie électronique, classique et à haute résolution, la sonde atomique tomographique, ou la diffraction et la spectrométrie d'absorption du rayonnement synchrotron [14].

La réussite de ces différents chantiers devrait permettre l'interpolation des résultats obtenus par les différents moyens d'irradiation neutronique ou par particules chargées, puis leur extrapolation à des conditions de réacteur de fusion.

\section{Le tungstène : matériau de protection et interaction avec le plasma}

Les matériaux de protection utilisés le plus communément à ce jour dans les tokamaks sont le graphite ou les composites carbone/carbone, et le béryllium dans le JET (Joint European Torus). Ils ont été sélectionnés pour leur compatibilité avec le plasma, que leur confere leur numéro atomique bas $(Z=4$ pour Be et $Z=12$ pour C). De plus, le graphite est réfractaire (point de sublimation : $3825^{\circ} \mathrm{C}$ ) et le béryllium est un bon "getter" des impuretés du plasma. Ils sont cependant écartés pour un futur réacteur de démonstration. En effet, les matériaux à base de carbone s'érodent fortement et les poussières qui en résultent ont une forte affinité chimique avec le tritium, conduisant à des inventaires de tritium incompatibles avec la sûreté. Le béryllium, quant à lui, a une température de fusion jugée trop basse $\left(\mathrm{T}_{\mathrm{F}}=1287^{\circ} \mathrm{C}\right)$ pour supporter les instabilités du plasma.

Finalement, le tungstène a été retenu sur la base de sa température de fusion, la plus élevée de tous les métaux $\left(\mathrm{T}_{\mathrm{F}}=3422^{\circ} \mathrm{C}\right)$, et de son taux d'érosion particulièrement faible. C'est aussi un élément à basse activation nucléaire. Son numéro atomique élevé $(Z=74)$ fait néanmoins que sa teneur dans le plasma devra être limitée à quelques $10 \mathrm{appm}$, alors que quelques pourcents atomiques de carbone pouvaient être tolérés. En effet, le rayonnement des impuretés contenues dans le plasma refroidit ce dernier et peut conduire à l'arrêt de la réaction de fusion. Pour les matériaux à forts $\mathrm{Z}$ comme le tungstène, le maximum de rayonnement se situe vers des températures électroniques de $\sim 1 \mathrm{keV}$, typiques du centre d'un plasma thermonucléaire, alors que pour ces mêmes températures, le rayonnement du carbone et du béryllium est inférieur de plusieurs ordres de grandeur. D'où la moindre tolérance du plasma thermonucléaire vis-à-vis du tungstène, et le fait que la cohabitation du plasma et du tungstène est un vrai problème..

Dans un réacteur de fusion, le tungstène, matériau de protection du divertor, sera fortement irradié et soumis à des flux élevés de particules $\alpha$ et de chaleur. Les projets WEST (voir encadré, p. 23, et note (1) ci-dessous) et ITER nous apprendront beaucoup en ce qui concerne la fabrication, l'intégration dans un tokamak, la tenue aux flux de particules et de chaleur, ainsi que la compatibilité avec le plasma. La simulation de flux de particules $\alpha$ d'énergie $\sim 50 \mathrm{eV}$, conduisant à des flux thermiques supérieurs aux $10 \mathrm{MW} / \mathrm{m}^{2}$ typiques d'ITER, amène des modifications significatives de la surface à l'échelle nanométrique, comme montré sur la figure 3 [15]. Comprendre et quantifier cet effet, l'érosion qui peut en résulter, ainsi que l'impact de la création simultanée de défauts ponctuels sur ces phénomènes, seront des enjeux majeurs des études de l'interaction entre un plasma de fusion DT d'un futur DEMO et le tungstène choisi comme matériau de protection.

\section{Validation et qualification des matériaux des réacteurs de fusion}

Il faudra qualifier les nouveaux matériaux développés pour les réacteurs de fusion.

Dans le cadre de l'approche élargie [1], l'Europe et le Japon étudient actuellement la conception et la validation expérimentale (EVEDA : Engineering Validation and Engineering Design Activities, en anglais) $\mathrm{du}$ projet de 1'International Fusion Materials Irradiation Facility (IFMIF), installation qui devrait simuler au mieux un spectre de neutrons de fusion et pourrait permettre l'obtention d'éléments de qualification des effets d'irradiation dans les matériaux de structure, avant le démarrage de DEMO.

Les neutrons d'IFMIF seront issus de réactions du type ${ }^{7} \mathrm{Li}+\mathrm{d} \rightarrow{ }^{7} \mathrm{Be}+2 \mathrm{n}$ et ${ }^{6} \mathrm{Li}+\mathrm{d} \rightarrow{ }^{7} \mathrm{Be}+\mathrm{n}$, créées par deux faisceaux de deutons (d) de $40 \mathrm{MeV}$ et d'une intensité de $125 \mathrm{~mA}$ chacun, impactant une cible de lithium liquide. Le spectre neutronique obtenu présentera un large pic autour de $14 \mathrm{MeV}$, permettant de reproduire les taux de déplacements atomiques, d'hélium et d'hydrogène typiques des réacteurs de fusion, avec trois volumes d'irradiation en fonction du débit de dose : 0,5 litre pour des forts flux (dose $>20 \mathrm{dpa} / \mathrm{an}), 6$ litres pour des flux moyens (1 à $20 \mathrm{dpa} / \mathrm{an})$, et plus de 8 litres pour les faibles flux (<1 dpa/an) [16].

L'équipe de l'EVEDA d'IFMIF est basée à Rokkasho au Japon. Elle devra remettre en 2017 un rapport qui contiendra une étude de conception détaillée de l'ensemble des installations d'IFMIF, une analyse de sûreté, ainsi que le bilan de la fabrication et des tests (i) d'un accélérateur prototype de $125 \mathrm{~mA}$ comprenant la source de deutons et les premiers étages d'accélération jusqu'à 9-10 MeV, et (ii) de la cible de lithium liquide. Les fortes intensité et accélération des accélérateurs, et la puissance élevée, $10 \mathrm{MW}$, de la cible au lithium liquide sont en effet les points durs de la faisabilité d'IFMIF. C'est à la suite de ce rapport que devrait pouvoir se faire le choix d'un site et se prendre la décision d'y construire IFMIF.

Ce type de source (fig. 4) permettra d'irradier des échantillons, puis de les tester en cellules blindées, ou bien d'effectuer des essais mécaniques in situ, notamment de fluage et de fatigue. Dû au volume disponible limité, un effort important a été fait pour développer des éprouvettes miniaturisées d'essais mécaniques, qui restent représentatives du comportement mécanique macroscopique [16].

La modélisation physique évoquée plus haut devra aussi contribuer à optimiser la matrice de tests dans IFMIF, à situer les résultats d'IFMIF par rapport à l'ensemble des connaissances et ainsi à les extrapoler de manière plus sûre aux conditions plus larges rencontrées dans les composants d'un réacteur.

Quant à la qualification des matériaux en conditions réelles, elle ne pourra se faire que dans le réacteur DEMO lui-même. C'est la démarche qui avait été suivie pour les matériaux de gainage de l'élément combustible des réacteurs rapides, développés en utilisant la simulation de l'irradiation aux neutrons par des irradiations par particules chargées, puis qualifiés par irradiation en réacteur.

(1) Le montage des premiers composants du divertor de WEST débutera fin 2014, avec un objectif de démarrage fin 2015-début 2016. 


\section{Conclusion}

Développer des matériaux de structure et de protection qui soient susceptibles de survivre aux conditions d'un réacteur de fusion constitue un défi majeur pour la communauté des métallurgistes et de la science des matériaux. Les laboratoires du CEA, du CNRS et de l'Université ont, en France, toutes les compétences et la plupart des outils nécessaires à la réussite d'une telle aventure. Le défi est sans doute plus grand que pour les structures de cœur des réacteurs de fission à neutrons rapides, avec, cependant, un atout considérable par rapport aux années 1970-90 : une modélisation physique, sur la base de calculs $a b$ initio, des effets d'irradiation, qui s'est révélée prédictive pour des alliages simples à base de fer et pour le tungstène, et dont un des défis majeurs sera la prédiction du comportement d'alliages industriels plus complexes.

\section{Remerciements}

Je voudrais remercier mes collègues du CEA et d'ITER pour leur relecture et commentaires critiques : Emmanuelle Tsitrone et Bernard Saoutic de l'Institut de Recherche sur la Fusion Magnétique, François Willaime du Département des Matériaux pour le Nucléaire, et Jean Jacquinot, conseiller scientifique auprès de l'Administrateur Général du CEA et expert auprès d'ITER.

\section{Références}

1- J. Jacquinot, Reflets de la physique, 32 (2013), 19-25.

2• J.L. Boutard, ce numéro, pp. 17-21.

3• www.efda.org/downloads/ppcs-overall-report

4 D. Maisonnier et al., Fusion Engineering and Design, 81 (2006) 1123-1130

5• www.cea.fr/recherche-fondamentale/fusionthermonucleaire-le-projet-west-au-cea-d

6• www.efda.org/efda/

7• L.L. Hsiung et al., Phys. Rev. B, 82 (2010) 184103.

8• Y. de Carlan, Les Clefs du CEA, n59, été 2010, p. 31.

9• J.L. Boutard et al., "ODS ferritic steels, a basic research joint program in France", soumis à J. Nucl. Mater.

10• J.P. Crocombette et F. Willaime, dans Comprehensive Nuclear Materials, edité par R. J. M. Konings, vol. 1, pp. 223-248 (Elsevier, 2012).

11• C.C. Fu et al., Nature Materials, 4 (2005) 68-74.

12• http://simap.grenoble-inp.fr/groupes-de-recherche/ simulations-par-dynamique-des-dislocations-discretes

13• J. Malaplate et al., Eng. Fract. Mech., 75 (2008) 3570-3580.

14• Y. Serruys et al., C.R. Physique, 9 (2008) 437-444.

15• G. de Temmerman et al., J. Nucl. Mater., 438 (2013) S78-S83.

16• A. Möslang, C.R. Physique, 9 (2008) 457-468. 